\title{
Tipo de cambio y crecimiento económico en México
}

\author{
Exchange rate and economic growth in Mexico \\ Verónica Cerezo García*
}

Universidad Nacional Autónoma de México, México

Recibido el 12 de febrero de 2020; aceptado el 22 de octubre de 2020

Disponible en Internet el: 23 de octubre de 2020

\section{Resumen}

Durante 2001-2018 la tasa de inflación se mantuvo baja y estable en México y el crecimiento económico registró una tasa promedio anual de $2.5 \%$, a pesar del constante aumento en las exportaciones. El notable estancamiento de la economía mexicana y de otras economías de América Latina, indujo a algunos autores a argumentar que el origen del lento crecimiento estriba en que los bancos centrales aprecian el tipo de cambio para anclar la inflación. Para acelerar el crecimiento económico proponen un tipo de cambio competitivo. Para comprobar esta hipótesis elaboramos un modelo de regresión con rupturas para calcular el tipo de cambio de equilibrio; a partir de esto estimamos un modelo NADRL para conocer las desviaciones (apreciaciones y depreciaciones) con respecto al tipo de cambio de equilibrio. Los resultados muestran que durante 1993-2018 un tipo de cambio estable y competitivo per se no acelera el crecimiento económico.

Código JEL: C10, F43, N16

Palabras clave: Tipo de cambio estable y competitivo; Crecimiento económico; Modelación econométrica; México

\footnotetext{
*Autor para correspondencia Correo electrónico: vcgcerezo@yahoo.com (V. Cerezo García).

La revisión por pares es responsabilidad de la Universidad Nacional Autónoma de México. 


\begin{abstract}
Mexico's rate of inflation has remained low and stable, while the average growth rate of output (2.5\%) has been stagnant over the period 2001-2018, despite the continuous expansion of exports. The persistent stagnation of the Mexican economy and other Latin American economies has prompted some authors to maintain that slow growth results from the Central Banks' practice of appreciating the exchange rate with the aim of anchoring inflation, hence the acceleration of economic growth depends on adopting a competitive exchange rate. In order to test this hypothesis, a regression model with breaks is used to estimate an equilibrium exchange rate; then, a NARDL model is estimated to determine exchange rate deviations (appreciations and depreciations) from the equilibrium value. It is empirically shown that a competitive and stable exchange rate per se was unable to accelerate output growth over the period 1993-2018.
\end{abstract}

JEL Code: $\mathrm{C} 10, \mathrm{~F} 43, \mathrm{~N} 16$

Keywords: Exchange rate; Economic growth; Econometric models; Mexico

\title{
Introducción
}

Con la crisis de 1995 en México se inició una transición para la estabilización de precios, objetivo que se alcanzó con el establecimiento de una meta de inflación. Durante el período 2001-2018 la tasa de inflación se ha mantenido estable en un rango de alrededor de 3\%. En paralelo, sin embargo, se observa la ralentización de la actividad económica expresada en el bajo crecimiento del PIB (en promedio anual $2.5 \%$ aproximadamente), a pesar del auge de las exportaciones.

El fenómeno de lento crecimiento se observa en otros países de América Latina. Para salir de la trampa del estancamiento y acelerar el crecimiento económico, algunos economistas han propuesto la adopción de una política de tipo de cambio competitivo. Luiz Carlos Bresser-Pereira $(2007,2011)$, uno de los autores de la teoría del Nuevo Desarrollismo, argumenta que el lento crecimiento se debe a la apreciación del tipo de cambio real; esto ha afectado la competitividad y provocado una menor rentabilidad de la inversión en el sector de bienes comerciables. Otros autores como Dani Rodrik (2008), Jaime Ros (2006, 2009, 2013), Razmi et al. (2012) y Roberto Frenkel (2006) también sostienen esta hipótesis: la apreciación del tipo de cambio es efecto de una política monetaria asimétrica ante movimientos de esa variable: la autoridad monetaria (el banco central o el Tesoro) es permisiva cuando el tipo de cambio se aprecia, pero interviene e impide el deslizamiento de la moneda cuando ésta se deprecia. Y esta política asimétrica deprime a la economía. La devaluación del tipo de cambio, sostienen, incrementa la competitividad de los bienes comerciables, propicia un aumento de las exportaciones y por ende de la tasa de crecimiento económico. Por tanto, argumentan que 
el tipo de cambio real debe alcanzar un nivel estable y competitivo a través de la política monetaria y cambiaria en conjunto (Ros y Galindo, 2006, 2009).

Existe un conflicto entre el modelo de crecimiento exportador y el modelo de metas de inflación en México, el cual se explica por el papel dual del tipo de cambio en la economía. El conflicto consiste en que el modelo exportador necesita un tipo de cambio competitivo y la estrategia de estabilidad de precios, basada en la regla de Taylor, necesita un tipo de cambio no competitivo. Si es así, para lograr la meta de inflación del Banco de México la apreciación del tipo de cambio es un requisito, lo cual sugiere que la inflación podría no ser un fenómeno puramente monetario, sino que tiene causas estructurales.

El objetivo principal del artículo es contrastar empíricamente la hipótesis nula que afirma que un tipo de cambio competitivo puede acelerar el crecimiento económico, tal como lo sostienen los autores mencionados.

El artículo se compone de la siguiente manera: primero discutimos teóricamente la relación del tipo de cambio con el nivel de los precios, la tasa de interés y la balanza de pagos. En la siguiente sección presentamos una revisión sucinta de la literatura empírica en lo que concierne a la estimación de modelos estructurales, las implicaciones que subyacen a la elección de régimen del tipo de cambio, los aspectos estructurales del tipo de cambio y la relación entre el tipo de cambio competitivo y el crecimiento económico. Más adelante, desarrollamos el análisis econométrico en el cual exponemos la metodología para calcular el tipo de cambio de equilibrio y sus respectivas desviaciones. El análisis empírico se enfoca en la relación entre el tipo de cambio y el crecimiento económico en México a través de la aplicación de un modelo NADRL para contrastar las hipótesis de que un tipo de cambio competitivo acelera el crecimiento económico versus la hipótesis que rechaza que esta sea la panacea del estancamiento estructural de la economía mexicana. En la conclusión resumimos los resultados de la discusión teórica y del análisis econométrico.

\section{Revisión de la literatura conceptual sobre tipo de cambio}

La teoría de la paridad del poder adquisitivo (PPA) plantea una relación de equilibrio entre las monedas con base en la inflación relativa; de esta forma ante un alza en la tasa de inflación inducida por vía nominal (aumento en la oferta monetaria) se requiere un ajuste en el tipo de cambio nominal en el largo plazo. Si se origina el proceso inflacionario ya sea del lado de la demanda o bien de la oferta, un ajuste en el tipo de cambio nominal provoca en la misma proporción un cambio en el tipo de cambio real que permite el retorno al equilibrio. Con base en este planteamiento, se ha afirmado que es necesario un régimen flexible para mantener un tipo de cambio competitivo que contribuya al crecimiento económico (Friedman, 1953; Bresser-Pereira, 2011, Frenkel, 2006; Ros y Galindo, 2006; Rodrik, 2008). 


\section{Tipo de cambio e inflación}

Partiendo de la PPA absoluta definida en la sección anterior, se puede expresar de forma general como sigue:

$$
e=\mathrm{P} * / \mathrm{P}
$$

La ecuación (1) muestra que el tipo de cambio real equivale a la razón de precios relativos; esto es, corresponde al nivel de precios nacional $\mathrm{P}$ con respecto al nivel de precios del extranjero $\mathrm{P}^{* 1}$. La ecuación representa un indicador del grado de competitividad internacional de los bienes comerciables de un país, dado que un aumento en $e$ expresa que los bienes comerciables nacionales son más competitivos que los extranjeros y también con respecto a los bienes no comerciables, y viceversa. La razón de precios relativos es un reflejo de los costos de producción para ambos bienes y del grado de competitividad que puede medirse a través de los costos unitarios de trabajo.

El PPA relativo que considera al tipo de cambio nominal E igual a los precios relativos multiplicado por una relación constante se puede expresar como

$$
\mathrm{E}=\varsigma \mathrm{P} * / \mathrm{P}
$$

Donde $\varsigma$ es un parámetro constante. Para el cálculo de la PPA relativa se utilizan comúnmente los índices de precios; sin embargo, es importante decir que en la ecuación (2) no existe una causalidad definida, de tal manera que la causalidad puede ser bidireccional, por lo que la razón de precios y E son variables endógenas. Por tanto, para comprobar la hipótesis de la PPA se utiliza la siguiente definición del tipo de cambio real $e$ :

$$
e=\mathrm{EP} * \mathrm{P}
$$

El tipo de cambio real es invariable en el tiempo t. A partir de la ecuación (3), entonces una depreciación (apreciación) del tipo de cambio nominal o real provoca un cambio en la asignación de recursos a favor (en contra) de los bienes comerciables nacionales. El tipo de cambio real expresado en la ecuación es un indicador de la competitividad internacional de un país; no obstante, no explica los cambios en la asignación de recursos entre ambos secto-

\footnotetext{
${ }^{1}$ La ratio de precios de bienes comerciables a precios de bienes no comerciables se utiliza comúnmente en el caso de países en desarrollo, mientras P/P* es un criterio que utiliza el Fondo Monetario Internacional. En nuestro caso, aplicamos el primer criterio que implica que una depreciación (apreciación) del tipo de cambio nominal y real se refleja en un incremento (decremento) de los respectivos índices.
} 
res (comerciables y no comerciables) ni los efectos de cambios en el sector externo y en la balanza de pagos, así como los impuestos al comercio exterior.

Se ha cuestionado la validez de la hipótesis de la PPA en el corto y en el largo plazo debido principalmente a la existencia de fuerzas externas que afectan el índice de precios. La hipótesis de la PPA se sostiene en las siguientes condiciones: i) los bienes comerciables o de exportación satisfacen la ley del precio único; ii) los bienes no comerciables o producidos localmente presentan la misma función de producción y los precios de los factores son equivalentes internacionalmente y iii) los bienes incluidos en el índice de precios se ponderan de la misma forma entre los países que comercian (Isard, 1995). Dadas estas condiciones, la validez de la PPA en el corto y largo plazos es difícil de sostener debido a que las características y estructuras de la producción, la dotación de los factores de producción y la composición de los índices de los precios no es uniforme entre los países.

No se ha encontrado evidencia suficiente a favor del cumplimiento de las condiciones ii) y iii) en el largo plazo. Algunos autores afirman que las desviaciones del PPA se han originado por choques monetarios más que por diferencias estructurales entre los países (Balassa, 1964). Los cambios en el índice de precios también se pueden explicar por la brecha de productividad (Samuelson, 1964), así como por la presencia de diferentes elasticidades ingreso de la demanda de importación y de exportación que afectan al tipo de cambio real (Houthakker y Magee, 1969). No obstante, en el caso de esta última evidencia, cuando se presenta en países con elasticidad ingreso de exportación mayor, se requiere redefinir la ecuación del tipo de cambio real, de suerte que disminuye su efecto adverso (Krugman, 1990). De esta forma, la hipótesis PPA puede ser válida en el largo plazo para el caso particular de los bienes exportables, si se satisfacen las condiciones restrictivas antes enunciadas.

Con respecto al comportamiento del tipo de cambio real, la evidencia empírica no valida la hipótesis de la PPA en el corto plazo, ya que la relación entre el tipo de cambio nominal y el nivel de precios no es estable. No obstante, aún con la presencia de estas desviaciones en el corto plazo e inclusive a mediano plazo no pueden invalidarse algunas teorías, como las de Samuelson y Balassa antes mencionadas. El efecto Balassa-Samuelson sobre el nivel de los precios que surge de factores estructurales para analizar el comportamiento de largo plazo y la validez de la hipótesis de la PPA fue objeto de análisis en la década de los años ochenta. El resultado de estos análisis afirma que se mantiene la hipótesis sólo para bienes comerciables y en el mediano plazo.

La evidencia muestra que la variabilidad del tipo de cambio real responde al régimen de tipo de cambio nominal (esto es, considerando una amplia banda entre los regímenes fijo y flexible), hecho que debe considerarse en la elección del régimen de tipo de cambio. En el caso de un régimen de tipo de cambio fijo las reservas internacionales son de gran importancia ante choques reales, por lo que al seleccionar un régimen de tipo de cambio fijo se aplican 
medidas opcionales que restringen el comercio exterior, como el control de capitales o bien impuestos que absorban o minimicen el choque real. En lo que concierne a un tipo de cambio flexible, la presión se ejerce en mayor medida sobre el tipo de cambio nominal. Se observa una mayor variabilidad del tipo de cambio real en la actualidad debido a la libre movilidad del capital internacional (Isard, 1995).

Ante la evidencia de una alta asociación entre el tipo de cambio nominal y el real es difícil afirmar que las variables nominales no tienen efecto alguno sobre las reales, como se sostiene en el enfoque neoclásico. Como contraparte, se argumenta que las fluctuaciones en el tipo de cambio nominal son resultado de choques reales y no de la política monetaria o de choques nominales. La presencia de precios "pegajosos o rígidos" eleva la importancia de usar al tipo de cambio como instrumento de política, dado que una depreciación nominal de la moneda puede generar mayor competitividad internacional si se deprecia el tipo de cambio real (Isard, 1995). A pesar de que la hipótesis de la PPA presenta límites teóricos y problemas para calcularse, en sentido práctico se utiliza como punto de partida para analizar el tipo de cambio real.

\section{Tipo de cambio y tasa de interés}

Con la adopción de la regla de Taylor como marco de la política monetaria, se hizo explícito el uso de la tasa de interés como instrumento para influir en la inflación y en el tipo de cambio: ante una apreciación (depreciación) del tipo de cambio la autoridad monetaria puede contrarrestarla disminuyendo (aumentando) la tasa de interés. Podemos observar la relación entre el tipo de cambio y las tasas de interés a través del proceso de compra y venta de moneda extranjera para realizar transacciones en el comercio internacional de bienes y servicios o bien en un portafolio de inversión internacional. La adquisición de moneda extranjera se puede realizar de manera inmediata en el mercado spot $(\mathrm{S})$ o bien por contrato en el mercado de futuros o forward (F). La teoría del tipo de cambio futuro explica que la distinción entre los tipos de cambio S y F se encuentra en el diferencial de las tasas de interés local y extranjera.

J.M. Keynes (1923) sistematizó la teoría del comportamiento del tipo de cambio futuro ${ }^{2}$ mediante lo que se conoce actualmente como la hipótesis de la paridad de tasas de interés. $\mathrm{Al}$ analizar la elección entre adquirir moneda extranjera en un centro financiero con respecto a otro, Keynes observó que mantener estos fondos dependía de varios factores, pero que el diferencial entre las tasas de interés local y extranjera era el más significativo. La hipótesis de la paridad de tasas de interés se conforma de la condición de paridad de interés cubierta (PIC) y la condición de paridad de interés no cubierta (PINC). Para explicar estas condiciones,

${ }^{2}$ En 1923 J.M. Keynes expuso su teoría completa en A Tract on Monetary Reform, resultado de varios suplementos publicados en The Manchester Guardian Commercial en 1922. 
supongamos que existen sólo dos monedas, X y Y, que la tasa de interés en el momento actual es $r_{t}$ y la tasa de interés futura $(t+1)$ del extranjero es $r^{*}$. Si un inversionista intercambia en el mercado spot una cantidad de la moneda X por la moneda Y entonces acumulará S (1 + rt) de moneda $Y$ en el tiempo $t+1$. En el caso de un arreglo de tipo de cambio futuro en el tiempo presente $\mathrm{t}$, el inversionista mantiene una cantidad de moneda $\mathrm{X}$ hasta el término del contrato futuro $\mathrm{t}+1$ en el que obtendrá $\mathrm{F}\left(1+\mathrm{r}^{*}\right)$ cantidad de moneda Y.

Entonces la hipótesis de la PIC se puede representar de la siguiente manera:

$$
\mathrm{Ft}-\mathrm{St} / \mathrm{St} \approx \mathrm{ft}-\mathrm{st} \approx \mathrm{r}-\mathrm{r}^{*}
$$

Donde f y s son los logaritmos del tipo de cambio forward y spot, respectivamente. La ecuación es una relación aproximada de la PIC donde el diferencial de las tasas de interés spot y forward son equivalentes a la diferencia de los logaritmos del tipo de cambio forward menos el spot. Esto implica que el premio que se obtiene de cambiar una moneda $\mathrm{S}$ o bien de un contrato a futuro $\mathrm{F}$ son aproximadamente equivalentes, dado que el inversionista paga por anticipado el riesgo o premio que recibirá en el periodo $t+1$.

En cambio, la condición PINC afirma que, si se mantiene una determinada cantidad de moneda $\mathrm{X}$ y posteriormente en un futuro se cambia por otra moneda $\mathrm{Y}$, no existe certidumbre de que cuando se cambie la moneda $X$ por moneda $Y$ en el periodo $t+1$ se obtendrá una ganancia. De esta manera, el inversionista tiene incertidumbre sobre el premio que obtiene hasta el momento futuro de conversión del tipo de cambio, esto es, entre el intervalo actual t y el futuro $t+1$. Es importante destacar que la hipótesis PINC supone que el mecanismo de las fuerzas del mercado por sí mismo asegura que el inversionista obtendrá el premio en $\mathrm{t}+1^{3}$.

La PINC se puede expresar como:

$$
\mathrm{EtSt}+1\left(1+\mathrm{rt}^{*}\right)=\mathrm{St}(1+\mathrm{rt})
$$

EtSt +1 es el valor esperado actual t del tipo de cambio spot en el tiempo $t+1$; entonces el diferencial del valor esperado del tipo de cambio spot y de las tasas de interés se puede representar como:

$$
\mathrm{EtSt}+1-\mathrm{St} \approx \mathrm{rt}-\mathrm{rt}^{*}
$$

\footnotetext{
${ }^{3}$ La condición de la PINC se basa en la teoría de mercados eficientes suponiendo que los agentes tienen información completa para la toma de decisiones. Esta condición se presenta en modelos de precios de activos internacionales.
} 
La ecuación (6) afirma que el diferencial entre el valor esperado y el actual del tipo de cambio spot es aproximadamente igual al diferencial presente entre las tasas de interés local y extranjera. Al cumplirse la ecuación (6) implica que el tipo de cambio spot esperado EtSt +1 puede pronosticarse con base en el diferencial de tasas de interés local y extranjera. La validez de ambas condiciones (PIC y PINC) tiene implicaciones en la intervención de los mercados cambiarios.

Con base en los supuestos de la PINC antes mencionados, si se comprueba su validez entonces la intervención de la autoridad no es eficaz y aun si no lo fuera se podrían aplicar instrumentos de política (como la tasa de interés) para alcanzar objetivos en el tipo de cambio $^{4}$. Por otra parte, con la validez de la PIC se acepta la intervención esterilizada para corregir las posibles desviaciones del tipo de cambio. Al considerar que las características en los mercados financieros presentan comportamientos similares con respecto al control de capital, al riesgo en el crédito y los impuestos y dado el supuesto de que la disponibilidad de instrumentos financieros sólo puede diferenciarse en la moneda en la cual se denominan, así como en las tasas de interés, entonces la validez de la PIC es más robusta. De esta forma, parece que la validez de la PIC puede cuestionarse sólo cuando ocurren choques financieros.

Se han utilizado principalmente dos fuentes para comprobar la validez de la PIC: su cálculo en la práctica y a partir del análisis de los datos. En el caso de los estudios empíricos que analizan los datos de la tasa de interés en varios países se han encontrado desviaciones que no han podido explicarse por factores como el riesgo político, los costos de transacción o el control de capitales. Por ello se sugiere seleccionar con cautela los datos de las muestras. La comprobación de la validez de la PINC con el supuesto que el diferencial en las tasas de interés puede pronosticar el tipo de cambio spot futuro (véase la ecuación 6), presenta la dificultad de encontrar datos para la variable EtSt +1 . Por ello, la calidad de los resultados de los pronósticos de la PINC se cuestiona.

Algunos análisis de regresión se centran en probar la validez de la PIC. No obstante, se ha obtenido una predicción sesgada y el coeficiente del diferencial de tasas de interés es negativo. Por consiguiente, considerando la evidencia anterior, no podemos afirmar que el diferencial de las tasas de interés $\left(\mathrm{rt}-\mathrm{rt}^{*}\right)$ pueda pronosticar las variaciones en el tipo de cambio. Esto cuestiona la influencia de los instrumentos de política monetaria sobre el comportamiento del tipo de cambio y su influencia tanto en variables nominales como reales.

\section{Tipo de cambio y la balanza de pagos}

Desde el siglo XVII se ha reconocido en los círculos políticos en Italia y en Gran Bretaña

\footnotetext{
${ }^{4}$ La intervención esterilizada en el mercado cambiario se realiza mediante la compra y venta de moneda extranjera para influir en el comportamiento del tipo de cambio.
} 
el papel del tipo de cambio en las desviaciones entre la balanza de pagos y las condiciones internas en la economía (Einzig, 1970). En la tradición de A. Marshall se considera el tipo de cambio como el precio que puede ajustar las desviaciones que surgen en la oferta y/o la demanda para volver al equilibrio. Este tipo de modelos se conoce como enfoque de elasticidades y se basa en los siguientes supuestos: se produce un bien importado $\mathrm{M}$ que se consume localmente (curva de demanda) y uno que se exporta (curva de oferta) X; los precios de ambos bienes se consideran en términos de la moneda local (PM y PX respectivamente); sólo se considera en el análisis el tipo de cambio nominal (razón unidad de moneda local a unidad de moneda extranjera) E; los mercados de bienes locales y de bienes exportados se equilibran; por último, se cumple la ley del precio único. El modelo explica que el equilibrio en la balanza comercial se puede lograr a través de los cambios en las elasticidades de la demanda y la oferta. Si se cumplen los supuestos antes mencionados, el modelo pronostica que una devaluación del tipo de cambio nominal E mejorará la balanza comercial sólo si la suma de ambas elasticidades de importaciones es mayor que uno. Al suponer que la oferta de las exportaciones es infinitamente elástica, la desigualdad antes mencionada se conoce como la condición Marshall-Lerner ${ }^{5}$. El enfoque de elasticidades analiza el efecto de las variaciones en el tipo de cambio nominal sobre la balanza de pagos considerando equilibrio parcial.

El enfoque de elasticidades presenta las siguientes limitaciones: sólo se ajusta el tipo de cambio nominal, de tal forma que al no incluir en el análisis los precios relativos omite el producto real y la capacidad productiva. No especifica el activo que se utiliza para realizar las transacciones de los bienes; por último, no considera que los cambios en la balanza comercial sean equivalentes a la cuenta del ingreso nacional (resultado del diferencial entre el producto y el gasto local) y estos cambios no se incluyen en el modelo.

En la década de los años cincuenta el enfoque de elasticidades se integró con la visión keynesiana del ingreso nacional. El resultado fue una fusión del enfoque elasticidades-absorción que sostiene que el ajuste en el tipo de cambio induce un cambio en la balanza de la cuenta corriente que, a su vez, tiene efectos en el empleo y la producción interna (Robinson, 1947; Harberger, 1950; Meade, 1951; Alexander, 1952). Según este esquema, una devaluación en la moneda local provoca una disminución en el precio del bien producido localmente que no sólo modifica la composición de la demanda, también eleva el nivel de producción que favorece a la balanza comercial.

Posterior al colapso del sistema de Bretton Woods en la década de los setenta, surgió nuevamente el interés en este enfoque elasticidades-absorción y en el manejo del tipo de cambio con base en este enfoque. Un ejemplo es la llamada curva J donde el efecto de una devaluación genera temporalmente un deterioro en la balanza comercial, dado que en el corto

\footnotetext{
${ }^{5}$ La condición Marshall-Lerner es un caso particular que emerge de la condición más amplia elaborada originalmente por Bickerdike (1920) y expuesta por Robinson (1947) y Metzler (1949).
} 
plazo el precio de las importaciones puede aumentar más rápidamente que el precio de las exportaciones. De esta forma, al inicio se experimenta un efecto negativo en la balanza comercial pero posteriormente ocurre un efecto positivo que provoca un superávit en la balanza comercial. Un argumento en contra del enfoque de elasticidades-absorción es que ofrece un análisis estático. Sin embargo, ha sido una premisa de las políticas de estabilización aplicadas en América Latina después de la crisis de deuda externa de 1982.

Los modelos antes mencionados sobre la balanza de pagos se centran en la relación del efecto del tipo de cambio sobre la cuenta corriente. No obstante, se omite el movimiento de los flujos de capital. En la década de los años sesenta surgió el modelo Mundell-Fleming que incluye en el análisis los flujos de capital privado y la cuenta corriente ${ }^{6}$. Similar al modelo IS-LM (Hicks, 1937), introduce el mercado de bienes y el mercado de dinero, con la diferencia que analiza una economía abierta suponiendo que el flujo de capital internacional neto depende de la tasa de interés local. El modelo considera los precios y la tasa de interés extranjera como exógenos; los instrumentos de política monetaria son la tasa de interés, el control de la oferta monetaria y el tipo de cambio. Por otra parte, el objetivo de la política fiscal es incrementar el ingreso para mantener el equilibrio presupuestario.

El modelo Mundell-Fleming explica el equilibrio de la balanza de pagos en términos de la igualdad entre el ingreso monetario y la tasa de interés (véase figura 1); considera dos casos de equilibrio de la balanza de pagos: la balanza comercial depende del ingreso local y la balanza de pagos general muestra la relación entre la balanza de la cuenta de capital y la tasa de interés local. En la figura 1 la balanza de pagos BP y el mercado de dinero D se representan con una curva con pendiente positiva, mientras que el mercado de bienes $\mathrm{B}$ se muestra con una curva descendente. Como podemos observar, el modelo Mundell-Fleming muestra el balance interno por medio de las curvas D y B; el balance externo corresponde a la balanza de pagos BP.

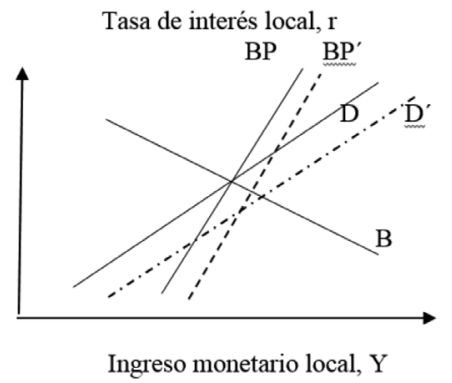

Figura 1. Modelo Mundell-Fleming.

Fuente: Elaboración propia

6 Los trabajos de Fleming (1962) y Mundell (1960, 1961a, 1961c, 1962 y 1963) se elaboraron independientemente. 
La figura 1 muestra que ante una devaluación de la moneda local la balanza de pagos y la curva $\mathrm{LM}$ se trasladan a la derecha $\left(\mathrm{BP}^{\prime}\right.$ y $\mathrm{D}^{\prime}$ respectivamente) mejorando la balanza comercial. En la figura 1 no se muestra de manera explícita el tipo de cambio, su efecto se ejerce a través del precio relativo de los bienes (local y extranjero) en las curvas BP y D. A cualquier nivel de ingreso, se puede aplicar una política monetaria de estabilización. En este caso, la disminución de la tasa de interés local propicia el equilibrio de la BP y del mercado de bienes. Sin embargo, si prevalece un régimen de tipo de cambio fijo la política monetaria por sí misma no puede lograr el equilibrio y el pleno empleo, se requiere utilizar la política fiscal $^{7}$. Como lo vislumbró Mundell (1963), el desafío es adoptar reglas para utilizar los instrumentos de política que aseguren los equilibrios interno y externo simultáneamente.

En el modelo Mundell-Fleming la aplicación de las políticas económicas (monetaria y/o fiscal) obedece al origen del desajuste (balance interno y/o externo), así como a su efectividad. Esta última, la efectividad de la política monetaria o fiscal, es resultado de las condiciones iniciales (régimen de tipo de cambio -fijo o flexible- y el grado de movilidad de los capitales). Con base en ello, la política monetaria y/o fiscal se orienta hacia un solo objetivo, ya sea corregir el desequilibrio interno o el externo; la intervención debe hacerse con el instrumento que ejerce una influencia directa y efectiva, tal como afirma el principio de mercado efectivo (Mundell, 1960). La efectividad de la política monetaria y fiscal depende del régimen de tipo de cambio y del grado de apertura de la cuenta de capitales que exista en la economía.

Mundell (1963) plantea dos escenarios: caso i) existe perfecta movilidad de capital y un régimen de tipo de cambio fijo, la aplicación de la política monetaria a través de un aumento en la oferta monetaria causa un alza en el volumen de las reservas de moneda extranjera, pero ningún efecto en el ingreso y el empleo. Para lograr un efecto positivo en el ingreso se requiere aplicar una política fiscal expansionista que, por otra parte, afecta negativamente a la balanza comercial. En el caso ii), existe movilidad de capital perfecta y tipo de cambio flexible. Un aumento de gasto público genera un cambio en la balanza comercial en el mismo sentido, pero ninguno en el ingreso y el empleo. En cambio, la expansión de la oferta monetaria puede dejar inalterada la tasa de interés, pero provoca una depreciación en el tipo de cambio favoreciendo a la balanza comercial y, por consiguiente, el ingreso y el empleo aumentan (Isard, 1995, pp. 102).

Sin embargo, los flujos de capital no son continuos, tal como supone el modelo Mundell-Fleming. El reconocimiento de este hecho condujo a analizar la relación entre el tipo de cambio y la balanza de pagos tomando en cuenta los cambios en el stock de la cuenta de

\footnotetext{
${ }^{7}$ Sobre la necesidad de aplicar políticas de estabilización, Mundell afirma que el principio de la teoría del comercio internacional clásica plantea la existencia de un mecanismo que asegura el equilibrio en la balanza de pagos que en realidad no se observa. De esta forma, la aplicación de la política monetaria es necesaria para lograr el pleno empleo desviándola de su función original mientras no se desarrolle un instrumento que pueda aplicarse a equilibrar la balanza de pagos (Mundell, 1961a).
} 
capital. A partir de ahí emergieron dos enfoques: el monetario y el de balance de cartera. La diferencia esencial entre ambos radica en que los activos financieros ( son sustitutos perfectos o imperfectos de activos del extranjero.

En la visión monetaria y en el balance de cartera el tipo de cambio se analiza en los llamados modelos de equilibrio de activos $^{8}$, los cuales parten de una perspectiva de equilibrio en el stock de activos y suponen que exclusivamente los residentes del país disponen de estos activos. Los modelos de balance de cartera se basan en el análisis de la demanda y la oferta del stock de cartera, incluyendo la tenencia de activos privados, así como los bonos gubernamentales que mediante su emisión pueden financiar el déficit fiscal. De este análisis se desprende que el stock de activos en la economía (dependiente de la política aplicada) es la variable que determina al tipo de cambio y la tasa de interés, las cuales se ajustan para mantener el mercado en equilibrio. No obstante, el sistema no se soluciona, ya que al incluir ambas $\mathrm{r}$ y r* no se determinan el tipo de cambio actual y el esperado.

\section{Análisis de la literatura empírica}

\section{Estimaciones empíricas de modelos estructurales}

Durante los años setenta y ochenta se realizaron diversos esfuerzos para explicar el comportamiento del tipo de cambio. Uno de esos enfoques utiliza los modelos estructurales de forma reducida basándose en tres modelos principalmente: el monetario con precios fijos, el monetario con precios flexibles y el de balance de cartera. Estos modelos tienen diferentes implicaciones de política económica. Un segundo enfoque es la aplicación de modelos estructurales de ecuaciones simultáneas o modelos macroeconométricos.

En términos generales, al estimar el tipo de cambio esperado a partir de las predicciones de ecuaciones de forma reducida, Meese y Rogoff (1983a; 1983b) encuentran varias limitaciones, a saber: sesgo por posibles variables omitidas, problema de simultaneidad y especificación errónea. Y aun corrigiendo el problema de simultaneidad y la inclusión de variables explicativas en términos reales persiste el problema. En su caso, los modelos de balance de cartera se han enfocado a comprobar si la prima de riesgo varía en el tiempo y si las intervenciones esterilizadas afectan al tipo de cambio (Meese y Rogoff, 1983a). Se puede concluir con base en la evidencia que se rechaza la hipótesis de no sesgo y el tipo de cambio presenta comportamientos no predecibles. Debido a algunos problemas como el de simultaneidad que surgen al estimar una ecuación de forma reducida, se ha explorado también la aplicación de modelos multivariados como alternativa. De estos últimos modelos se puede mencionar que estiman

${ }^{8}$ El desarrollo de estos modelos se suscitó en la década de los años setenta, después del colapso del sistema Bretton Woods; su desarrollo también se relaciona con el avance del análisis econométrico. 
con precisión los parámetros; no obstante, presentan problemas de especificación errónea que se originan en una ecuación y pueden afectar al resto de ecuaciones. Podemos concluir que a pesar de algunas limitantes que presentan los modelos de forma reducida y multivariados, en la práctica se utilizan para el cálculo del tipo de cambio y obtienen resultados satisfactorios.

\section{Selección del régimen de tipo de cambio: fijo versus flexible}

La relación entre el tipo de cambio y las variables económicas (tasas de interés, balanza de pagos, nivel de precios, crecimiento económico, empleo, etc.) obedece a los objetivos de política económica de las autoridades. La selección del régimen de tipo de cambio puede ser un esquema rígido o flexible o alguno intermedio entre ambos regímenes extremos ${ }^{9}$.

En el caso de un régimen de tipo de cambio rígido, el gobierno asigna una paridad cambiaria determinada, que mantiene constante mediante la compra o venta de moneda extranjera. El banco central realiza intervenciones oficiales en el mercado cambiario, de esta forma la compra (venta) de moneda extranjera aumenta (disminuye) las reservas. Como la apreciación y depreciación son nulas, entonces la condición de paridad de la tasa de interés es válida $\left(r=r^{*}\right)$.

En un régimen de tipo de cambio flexible no hay intervención del gobierno o del banco central en el mercado cambiario. Por tanto, el tipo de cambio se determina por la oferta y la demanda de la moneda nacional con respecto a otras monedas. Como vimos, en este caso se considera válida la PINC, ya que el arbitraje determina las variaciones en el tipo de cambio.

El comportamiento del tipo de cambio también responde al sistema monetario internacional que se establece por convención en la mayoría de los países. Su objetivo es monitorear y manejar el tipo de cambio entre diferentes monedas. El sistema Bretton Woods (1946-1971) se caracterizó principalmente por ser un período que se inclinó a un régimen de tipo de cambio fijo, inflación estable y con altas tasas de crecimiento económico. Posteriormente a Bretton Woods el régimen de tipo de cambio flexible se ha propagado rápidamente, sobre todo a partir de la década de los años noventa, resultado del desarrollo financiero y de la creciente movilidad del flujo de capitales. De lo anterior se desprende que una de las funciones del tipo de cambio es mantener el equilibrio interno y externo en la economía. Por tanto, la elección del régimen tiene importantes implicaciones de política económica. Para el caso de un régimen de tipo de cambio fijo la política monetaria se enfoca en mantener la estabilidad por medio de intervenciones en el mercado cambiario. Por el contrario, con un régimen de tipo de cambio flexible la política monetaria es autónoma y se puede orientar a otros objetivos internos.

Las ventajas y desventajas que deben contemplarse al adoptar un régimen fijo o flexible

${ }^{9}$ El Fondo Monetario Internacional ofrece una clasificación oficial del tipo de cambio: fijo, flexible y un régimen intermedio entre ambos con flexibilidad limitada. 
(o alguno intermedio) fueron discutidas desde la década de los años sesenta ${ }^{10}$. El enfoque monetarista (Friedman, 1953; Frenkel, 1981) argumenta en contra del tipo de cambio fijo (debido al alto costo de mantenerlo estable) y a favor del tipo de cambio flexible, ya que puede ajustarse para mejorar la competitividad ante precios y salarios internacionales rígidos y estabilizar la especulación de capital privado $^{11}$.

En el análisis de Mundell (1961b) la selección óptima de un régimen de tipo de cambio debe contemplar los siguientes aspectos: las características estructurales ${ }^{12}$, el ambiente externo y macroeconómico que cambia de forma constante y los flujos de capital. Nurkse (1944) cuestionó que el libre ajuste del tipo de cambio contribuya al equilibrio de la balanza comercial y que mantenga la estabilidad ante los movimientos de capital. De hecho, considera un régimen donde el tipo de cambio es volátil. Krugman, (1989), a su vez, afirma que el tipo de cambio flexible puede ser adverso a la inversión productiva cuando se encuentra apreciado; y que el efecto de una depreciación puede ser negativo para la creación de capacidad productiva y para las ganancias del capital.

Por otra parte, la adopción de un régimen de tipo de cambio fijo durante el sistema Bretton Woods se caracterizó por un crecimiento económico estable. Durante la década de los años noventa caracterizada por la mayor apertura comercial y financiera, los estudios empíricos realizados por el Fondo Monetario Internacional para el periodo 1980-2006 concluyen, en términos generales, que los países en desarrollo deben adoptar regímenes intermedios, es decir, entre el tipo de cambio peg $^{13}$ y el flotante. La elección del tipo de cambio es importante, dado que puede afectar el entorno macroeconómico (el crecimiento, la inflación), así como al sistema monetario internacional. La evidencia ha mostrado que al adoptar un tipo de cambio rígido la autoridad pierde capacidad y libertad para aplicar políticas macroeconómicas. Por el contrario, en un régimen de completa flexibilidad el nivel de precios relativos se ajusta, pero también puede conducir a desviaciones del tipo de cambio y choques de corto plazo. De esta forma, un tipo de cambio estable depende de la productividad, la disciplina fiscal y monetaria, del control en el flujo de capitales y de la cooperación política internacional (Isard, 1995).

\footnotetext{
${ }^{10}$ Originalmente el debate se centró en discutir si los flujos de capital internacionales podrían estabilizarse mediante un régimen de tipo de cambio flexible.

${ }^{11} \mathrm{El}$ argumento que el tipo de cambio se puede ajustar para evitar períodos inflacionarios y deflacionarios se sostiene en la validez de la PPA donde el tipo de cambio real equivale al diferencial de inflación $\left(\mathrm{P}-\mathrm{P}^{*}\right)$

${ }^{12}$ Como características estructurales se consideran el tamaño y la apertura comercial, la diversificación de las actividades productivas y las habilidades de la fuerza de trabajo, la movilidad geográfica de factores productivos, el mecanismo de redistribución fiscal, las preferencias de política, la flexibilidad de precios y la tasa de salarios, la exposición a choques locales y externos y el desarrollo financiero.

${ }^{13}$ El término tipo de cambio peg se refiere a cuando una moneda se vincula con otra en busca de estabilidad. Para lograrlo las autoridades deben mantener grandes reservas de la moneda a la cual está vinculada la local para controlar las variaciones en la oferta y la demanda en el mercado cambiario.
} 


\section{Condiciones estructurales del tipo de cambio}

Las distintas teorías explican que la determinación del tipo de cambio obedece a diversos factores: la inflación relativa en el caso de la hipótesis PPA; la demanda en el caso del modelo de elasticidades-absorción; el enfoque de portafolios enfatiza el mercado de divisas y de activos financieros y el enfoque monetarista lo explica a partir de las ofertas relativas de dinero.

La teoría estructuralista explica el tipo de cambio a partir de las condiciones estructurales de la economía; el tipo de cambio es un fenómeno estructural, no monetario. Y su dinámica obedece a los costos de producción, la productividad y las restricciones estructurales de la balanza de pagos. Desde este punto de vista, la inestabilidad del tipo de cambio es una consecuencia, no una causa, de los problemas de la balanza de pagos, de las restricciones estructurales y externas que limitan el crecimiento económico. Estas condiciones estructurales que, para el caso de economías como la mexicana, se resumen en la Ley de Engel y en la llamada Ley de Thirlwall, significan que los desequilibrios de la balanza en cuenta corriente se deben al diferencial adverso entre las elasticidades ingreso de la demanda de exportaciones y de importaciones. Esto determina un déficit estructural y endémico de la balanza de pagos que debe financiarse con un superávit en la balanza de capitales.

Los flujos de capital ejercen el efecto apreciación de la moneda de las economías deficitarias. De esta forma, la tendencia a la apreciación del tipo de cambio que observamos es consecuencia de los problemas estructurales de la economía. Por tanto, esta apreciación no es la causa del estancamiento económico, sino el efecto de la dinámica de una economía cuyo crecimiento confronta la asimetría entre las elasticidades ingreso antes aludidas (la Ley de Engel) y, por ello, se halla restringido por el equilibrio de la balanza de pagos (la Ley de Thirlwall) (véanse Perrotini y Vázquez, 2017 y 2018; Perrotini, Vázquez y Angoa, 2019).

\section{El tipo de cambio real competitivo y el crecimiento económico}

Varios autores proponen a la política de tipo de cambio competitivo como instrumento para incentivar el crecimiento, en especial en los países en desarrollo. En una economía que sigue un modelo orientado a las exportaciones, esta política puede generar un efecto comercio (aumento de las exportaciones y detrimento de las importaciones), un efecto pecuniario (aumento del ingreso de divisas, de los hogares y las empresas) y un efecto desarrollo (las empresas con rendimientos crecientes se benefician del acceso a la tecnología). Los efectos se logran a través de tres mecanismos: disminución de los salarios, el canal macroeconómico y el de desarrollo (Dvoskin y Feldman, 2015) ${ }^{14}$

\footnotetext{
${ }^{14}$ Los mecanismos mencionados presuponen la existencia de una alta elasticidad salario ante cambios en la demanda de trabajo, la absorción completa de la demanda de exportaciones (condición Marshall-Lerner), la demanda
} 
La evidencia empírica muestra que la devaluación del tipo de cambio tiene un efecto positivo en el crecimiento, dado que aumentan las exportaciones y fortalece el sector de bienes comerciables o industrial en la economía. Existen varios estudios como el de Rodrik (2008) que analiza el período 1950-2004 con datos panel de 188 países y elabora un índice de depreciación -o sobrevaluación- con base en el índice de precios ajustado por el efecto Samuelson-Balassa. Demuestra que la política cambiaria puede funcionar como un "second best" ante los costos de distorsión en la economía. ${ }^{15}$ Ros y Galindo (2006) muestran la existencia de una relación positiva entre el tipo de cambio y crecimiento para el caso de México al evaluar el papel de la política monetaria y cambiaria para lograr la meta de inflación. Encuentran una asociación positiva entre el tipo de cambio real y la inversión, por tanto, concluyen que una devaluación del tipo de cambio real genera crecimiento económico en el largo plazo.

\section{Discusión de la literatura empírica}

El comportamiento del tipo de cambio real en el tiempo es inestable debido a que depende de varios factores, por ejemplo, la productividad, el gasto del gobierno, los términos de intercambio, las medidas no arancelarias, el balance de activos extranjeros y los flujos de capital que influyen en el nivel de precios y el tipo de cambio nominal. Asimismo, después de la caída del sistema monetario y financiero internacional de Bretton Woods la discusión se ha centrado en la importancia del tipo de cambio nominal como instrumento estratégico para mantener la estabilidad o aliviar la inestabilidad. De esta forma, la elección del régimen y sus implicaciones en las economías en desarrollo es un tema de debate actual que requiere definir y calcular un tipo de cambio de equilibrio como base comparativa para evaluar el costo-beneficio de la política cambiaria adoptada por las autoridades monetarias.

También identificamos que existe un paradójico consenso entre algunos autores neoclásicos (teoría de la PPA y enfoque monetarista) y heterodoxos acerca de las virtudes del tipo de cambio para acelerar el crecimiento. Ante un alza en la tasa de inflación (aumento de la oferta monetaria) se requiere un ajuste del tipo de cambio nominal en el largo plazo que provoca un ajuste equivalente en el tipo de cambio real con el que se retorna al equilibrio. Por tanto, es indispensable un régimen de tipo de cambio flexible que mantenga un tipo de cambio competitivo que ejerce una mayor presión en el tipo de cambio nominal. ${ }^{16}$

de inversiones no presenta problemas de realización y retornos uniformes de capital ante apertura de los flujos de capital.

${ }^{15}$ Debido a la dificultad en la medición de las fallas de mercado, Rodrik (2008, p. 397) afirma que es prácticamente imposible proveer evidencia directa de que algunos tipos de bienes presenten esta clase de distorsiones.

${ }^{16}$ Debido a la asociación entre el tipo de cambio nominal y real no se puede afirmar que las variables nominales no ejercen efecto sobre las reales, como sostiene el monetarismo. 
El cálculo del tipo de cambio real de equilibrio (TCRE) sirve como referencia para conocer si una moneda se encuentra sobrevaluada o subvaluada. ${ }^{17}$. Williamson (1985) propone calcular el TCRE utilizando el tipo de cambio de equilibrio fundamental (FEER). La necesidad de estimar el TCRE consistente con el desempeño macroeconómico es importante en el esquema de política económica del Consenso de Washington debido a su influencia en el crecimiento. A partir de este enfoque, se considera el tipo de cambio apropiado para la estabilidad macroeconómica y la competitividad que incentive las exportaciones y mantenga el equilibrio externo. De esta forma, cualquier desviación del TCRE 'apropiado' puede perjudicar al crecimiento económico (Williamson, 1985).

Se estima el FEER aplicando modelos econométricos estructurales sustentados en variables económicas 'fundamentales'. John Williamson $(1985,1994)$ afirma que el cálculo del FEER es adecuado para calcular el tipo de cambio de equilibrio que sirve como referente. El FEER se define como el tipo de cambio real efectivo en equilibrio consistente con el balance macroeconómico; esto supone una economía en pleno empleo y baja inflación (balance interno) en conjunto con una cuenta corriente sostenible o en concordancia con los flujos de capital (balance externo). El término 'fundamentales' considera sólo efectos de las variables en el mediano y largo plazos excluyendo los de corto plazo (Clark y MacDonald, 1998). Debido a que el FEER varía a través del tiempo, se busca que el tipo de cambio real de equilibrio tenga como objetivos alcanzar el crecimiento del producto potencial, una cuenta corriente sostenible y que, con base en las variaciones del tipo de cambio real efectivo, pueda conocerse su trayectoria (Williamson, 1994).

El cálculo del FEER se realiza a través de la estimación de patrones de equilibrio del tipo de cambio real con base en la especificación (o supuesto) del comportamiento de los 'fundamentales' y las ecuaciones del tipo de cambio real (Elbadawi, 1994). De esta forma, el FEER ofrece un enfoque del tipo de cambio real efectivo a partir de los fundamentales que puede representarse por la igualdad entre la cuenta corriente y la de capitales. La complejidad para calcularlo radica en el número de parámetros que es necesario incluir en el modelo de estimación.

\section{Análisis econométrico}

Proponemos un modelo econométrico estructural para estimar el TCRE como una aproximación al FEER. A partir del TCRE podremos conocer las desviaciones del tipo de cambio real (apreciaciones y depreciaciones) y su efecto en el crecimiento económico en México.

\footnotetext{
${ }^{17}$ EL TCRE se refiere al equilibrio entre los balances interno y externo en una economía (Nurkse, 1945), que varía, de acuerdo con la hipótesis PPA, debido a factores reales y nominales a corto plazo y sólo reales a largo plazo (Edwards, 1991).
} 
De esta forma, el análisis econométrico se centra en comprobar la validez del tipo de cambio competitivo y estable.

La hipótesis nula supone que devaluar el tipo de cambio ejerce un efecto positivo en el crecimiento económico y la hipótesis alternativa supone que ejerce un efecto negativo. La metodología aplicada es la siguiente: primero realizamos las pruebas de raíces unitarias (Prueba Aumentada Dickey-Fuller y Phillips-Perron) de las variables en niveles y en diferencia para conocer su orden de integración. Segundo, aplicamos la prueba de cointegración Engel-Granger (1987). Si existe una relación de largo plazo entonces estimamos el TCRE. Para estimarlo realizamos una regresión de mínimos cuadrados con rupturas seleccionadas aplicando la prueba Bai-Perron (2003). Tercero, eliminamos los efectos de corto plazo y aleatorios mediante el filtro Hodrick-Prescott a partir de los datos estimados. Por último, aplicamos un modelo NADRL para comprobar la validez de la hipótesis nula antes mencionada.

El modelo NADRL tiene la ventaja de estimar al mismo tiempo la asimetría y la cointegración de variables no estacionarias en el largo plazo expresado de la siguiente manera:

$$
\Delta \mathrm{Y}_{\mathrm{t}}=\gamma+\beta_{\mathrm{y}} \mathrm{Y}_{\mathrm{t}-1}+\beta_{X} \mathrm{X}_{\mathrm{t}-1}+\sum_{\mathrm{i}=1}^{\mathrm{p}-1} \delta_{\mathrm{i}} \Delta \mathrm{Y}_{\mathrm{t}-1}+\sum_{\mathrm{i}=0}^{\mathrm{q}-1}\left(\omega_{\mathrm{i}}^{+} \Delta \mathrm{X}_{\mathrm{t}-1}^{+}+\omega_{\mathrm{i}}^{-} \Delta \mathrm{X}_{\mathrm{t}-1}^{-}\right)+\square_{\mathrm{t}}
$$

$\gamma$ es una constante, Yt y Xt las variables dependiente e independiente, respectivamente, ঠi corresponde a los rezagos de la variable dependiente en el período i, p y q denotan el número de rezagos distribuidos de las variables dependientes y exógenas, respectivamente, $\Delta$ es el operador que denota variaciones en el crecimiento, wi son los coeficientes positivos y negativos de la variable independiente y, por último, et es el término de error en el tiempo t. A través de la inclusión de variables independientes positivas y negativas se deriva un modelo de corrección de error dinámico con el cual se pueden estimar las asimetrías de corto $\left({\omega_{i}^{+}}^{+}\right.$y ${\omega_{i}^{-}}^{-})$ y largo plazo ( $\beta^{\prime}$ s), respectivamente y su efecto en la variable dependiente.

El análisis se realiza para una muestra que consta de 104 observaciones con datos trimestrales correspondientes al período 1993Q1-2018Q4 para el caso de México. Las variables económicas fundamentales (FEER) que se incluyen en el análisis son: la oferta monetaria por su efecto a corto plazo; los términos de comercio; las reservas internacionales y la razón de precios de bienes comerciables a no comerciables ${ }^{18}$, por su influencia a largo plazo. Los términos de intercambio se obtienen de la razón entre el índice de precios de las exportaciones con respecto al de las importaciones; las reservas internacionales se encuentran denominadas en dólares e indican cuando aumenta o disminuye la demanda de moneda local; la razón de

\footnotetext{
${ }^{18}$ Los precios de los bienes comerciables se calculan considerando actividades primarias, minería y manufacturas; el índice de precios de los bienes no comerciables incluye actividades de construcción, servicios de energía eléctrica, agua, gas y terciarios. Los datos proceden del INEGI (Instituto Nacional de Estadística, Geografía e Informática).
} 
precios es un indicador de competitividad y la oferta monetaria indica los ajustes resultantes de la política monetaria.

Procedemos a especificar el modelo econométrico para calcular el TCRE de la siguiente manera:

$$
\mathrm{TCRE}=\mathrm{C}+\alpha 1 R E S+\alpha 2 M O N+\alpha 3 T N T+\alpha 4 T O T+\varepsilon
$$

Donde RES son las reservas internacionales denominadas en moneda extranjera, $M O N$ es la oferta monetaria, TNT es la razón de precios de bienes comerciables a no comerciables, TOT son los términos de intercambio y $\varepsilon$ representa el término de error. Incluimos las variables que afectan al TCRE en el corto y largo plazo. Como vemos, el modelo incluye variables que representan la cuenta corriente (balanza comercial y términos de intercambio) y la cuenta de capitales (reservas en moneda extranjera y oferta monetaria).

A través del análisis de integración de las variables en niveles y en primera diferencia se aplican las pruebas Dickey-Fuller aumentada (DFA) y Phillips-Perron (PP). Se comprueba que las variables son estacionarias en primera diferencia y seleccionamos el número de rezagos óptimo con el criterio Akaike (ver Tabla A1 en el anexo). También se encuentra que las variables presentan una relación de largo plazo con la prueba de Engel-Granger y se comprueba la estabilidad en los parámetros con la prueba de Hansen (1992) que acepta la hipótesis nula: las series están cointegradas (ver Tabla A2 en el anexo).

La serie en el período de análisis presenta varios cambios estructurales que se comprueban con la prueba secuencial Bai y Perron (2003), la cual determina las rupturas que deben incorporarse en la muestra (véase tabla 1). Con el estadístico F y un nivel de significancia del 5\% se determina como cuatro el número de las rupturas óptimas. De esta forma, procedemos a estimar el TCRE a través de una regresión de mínimos cuadrados con intercepto e incluyendo 4 rupturas (1995Q4, 1999Q2, 2009Q3, 2015Q1) segmentando la serie completa en cinco partes.

Tabla 1

Prueba múltiple de rupturas Bai-Perron

\begin{tabular}{cccc}
\hline $\begin{array}{c}\text { Prueba de } \\
\text { ruptura }\end{array}$ & F-estadístico & $\begin{array}{c}\text { Escala } \\
\text { F-estadístico }\end{array}$ & $\begin{array}{c}\text { Valor } \\
\text { Crítico** }\end{array}$ \\
\hline 0 vs. $1 *$ & 36.54 & 182.69 & 18.68 \\
1 vs. $2 *$ & 22.66 & 113.31 & 20.57 \\
2 vs. $3 *$ & 20.58 & 102.89 & 21.60 \\
3 vs. $4 *$ & 27.61 & 138.07 & 22.55 \\
4 vs. 5 & 3.74 & 18.70 & 23.00 \\
\hline
\end{tabular}

Nota: Nivel de significancia al 5\%.

Fuente: elaboración propia. 
Los resultados de la regresión con rupturas para estimar el TCRE se presentan en la tabla 2.

Tabla 2

Modelo con regresión de rupturas

Variable dependiente : TCRE

Variable

MON

RES

C

1999Q2 - 2009Q2 -- 41 obs

TOT

TNT

MON

RES

$\mathrm{C}$

2009Q3 - 2014Q4 -- 22 obs

TOT

TNT

MON

RES

C

2015Q1 - 2018Q4 -- 16 obs

TOT

TNT

MON

RES

$\mathrm{C}$

R Cuadrada

$\mathrm{R}$ cuadrada ajustada

Error de desviación

\section{Coeficiente}

$-1.58 \mathrm{E}-08$

4.09E-05

$-308.25$

333.60

1.02

7.50E-10

1.59E-05

$-342.18$

455.64

0.99

$-4.90 \mathrm{E}-09$

6.53E-05

$-450.56$

689.75

0.95

$-1.75 \mathrm{E}-10$

8.89E-06

$-659.62$

0.99

0.99

0.77

Nota: Nivel de significancia 5\%; se determinan las rupturas con la prueba Bai-Perron y SRC es la suma de residuo al cuadrado.

Fuente: Elaboración propia.
t-Estadístico Prob.

$-0.69 \quad 0.49$

$0.18 \quad 0.86$

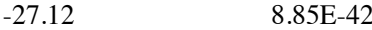

$80.47 \quad 1.44 \mathrm{E}-77$

$183.64 \quad 1.03 \mathrm{E}-105$

$\begin{array}{ll}0.35 & 0.73\end{array}$

$\begin{array}{ll}0.56 & 0.58\end{array}$

\begin{tabular}{l}
$-62.84 \quad 3.25 \mathrm{E}-69$ \\
\hline
\end{tabular}

$33.93 \quad 7.43 \mathrm{E}-49$

$100.48 \quad 4.08 \mathrm{E}-85$

$\begin{array}{ll}-1.53 & 0.13\end{array}$

$\begin{array}{ll}1.60 & 0.11\end{array}$

$-28.70 \quad 1.54 \mathrm{E}-43$

$30.71 \quad 1.12 \mathrm{E}-45$

$107.11 \quad 2.72 \mathrm{E}-87$

$\begin{array}{ll}-0.13 & 0.89\end{array}$

$\begin{array}{ll}0.18 & 0.86\end{array}$

$-24.79$

$5.11 \mathrm{E}-39$

SRC

47.35

F-estadístico

148175.48

Prob(F-Estadístico) 
Al comparar en el período de análisis el TCRE con el crecimiento económico, se observa una fuerte desviación en la que el tipo de cambio se encuentra por debajo del TCRE estimado (depreciación) sin impulsar el crecimiento a partir del 2014 (véase figura 2).

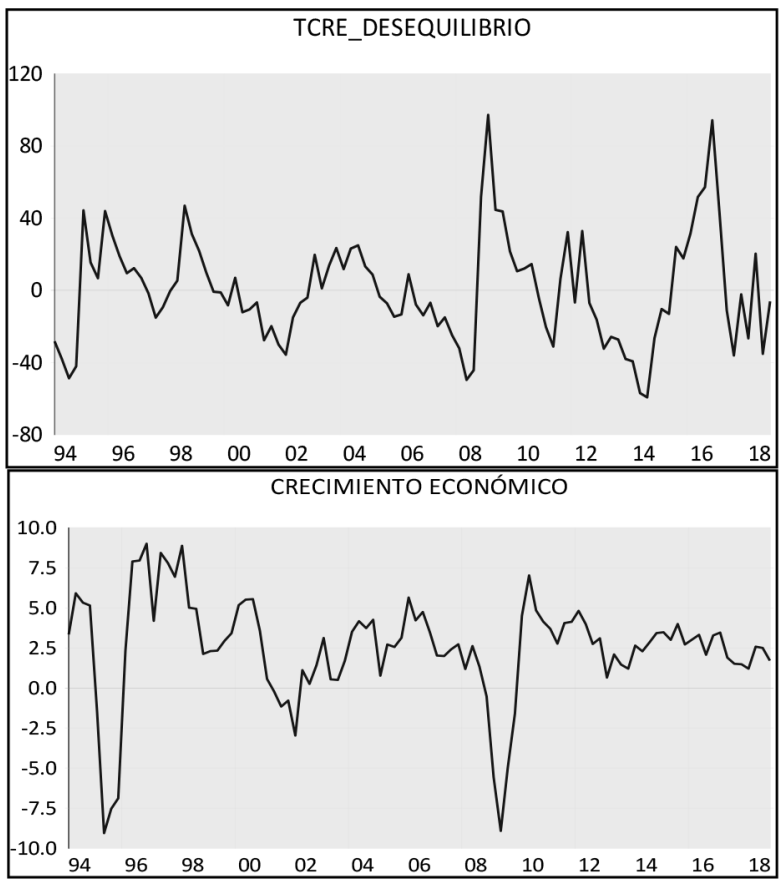

Figura 2. El TCRE estimado y el crecimiento económico (GY).

Fuente: Elaboración propia.

La tabla 3 muestra el modelo seleccionado ADRL donde la variable dependiente es el crecimiento del producto (GY) y la variable independiente es el TCRE estimado anteriormente.

El modelo seleccionado $(5,3,0)$ a través del criterio de información Hanna-Quinn (HG) incluye cinco rezagos de la variable dependiente correspondiente a la tasa de crecimiento (GY), tres rezagos TCRE POS(-3) y un rezago TCRE NEG(-1).

Aplicamos las pruebas LM de autocorrelación serial Breusch-Godfrey para cuatro rezagos sin rechazar la hipótesis nula de correlación serial. La prueba de Breusch-Pagan-Godfrey rechaza la presencia de heterocedasticidad (véase Tabla A3 en el anexo).

En los resultados del modelo se observan los efectos de una apreciación y una depreciación del TCRE en el crecimiento económico. De acuerdo con el enfoque nuevo desarrollista y la hipótesis nula, una devaluación podría mejorar la competitividad y aumentar las exportacio- 
nes, lo que provocaría un efecto positivo en el crecimiento. Al contrario, una apreciación del tipo de cambio generaría un efecto negativo. Encontramos que todos los coeficientes, tanto positivos como negativos, son significativos, excepto el TCRE (POS). De hecho, el primer rezago provoca un efecto negativo en el crecimiento, y desaparece rápidamente en el segundo y el tercer trimestre. Esto puede implicar que el efecto es de corto plazo o a lo sumo de mediano plazo. En el caso de una depreciación sólo se incluye el primer rezago TCRE (NEG), que es significativo y positivo; no obstante, el impacto en el crecimiento es muy pequeño y de corto plazo en el primer trimestre. Esto es, una depreciación de $1 \%$ provoca un aumento en el crecimiento de apenas 0.0176 en el crecimiento económico.

Tabla 3

Modelo NADRL: Crecimiento-TCRE

\begin{tabular}{lccc}
\hline \multicolumn{2}{l}{$\begin{array}{l}\text { Variable Dependiente: Crecimiento Económico GY } \\
\text { Modelo Seleccionado: NARDL(5,3,0) }\end{array}$} & & \\
\hline Variable & Coeficiente & t-Estadístico & Prob.* $^{*}$ \\
\hline GY(-1) & 0.76 & 8.11 & 0.00 \\
GY(-2) & 0.19 & 1.70 & 0.09 \\
GY(-3) & -0.26 & -2.38 & 0.02 \\
GY(-4) & -0.37 & -3.29 & 0.00 \\
GY(-5) & 0.39 & 4.71 & 0.00 \\
TCRE_DESEQUILIBRIO_POS & -0.01 & -1.15 & 0.25 \\
TCRE_DESEQUILIBRIO_POS(-1) & -0.03 & -1.88 & 0.06 \\
TCRE_DESEQUILIBRIO_POS(-2) & 0.04 & 2.65 & 0.01 \\
TCRE_DESEQUILIBRIO_POS(-3) & 0.02 & 1.93 & 0.06 \\
TCRE_DESEQUILIBRIO_NEG & 0.02 & 2.29 & 0.02 \\
C & 0.83 & 1.80 & 0.08 \\
\hline R-cuadrada & 0.80 & SRC & 207.89 \\
R-cuadrada Ajustada & 0.78 & F-Estad. & 34.02 \\
Error de desviación & 1.57 & Prob. & 0 \\
\hline
\end{tabular}

Nota: Selección del modelo por el criterio de Hannan-Quinn (HQ).

Fuente: Elaboración propia.

\section{Conclusión}

Como fruto de la revisión teórica se identificaron aspectos en los cuales existe consenso entre la teoría neoclásica (Hipótesis de la PPA y el monetarismo) y los autores que promueven una 
política cambiaria competitiva. Desde este enfoque es necesario mantener un régimen de tipo de cambio flexible y estabilidad en los precios; como no se cumple la condición PINC se observa que el tipo de cambio se aprecia y la tasa de interés no lo controla; por último, su análisis se centra en el efecto que ejerce la depreciación en la cuenta corriente sin considerar los que afectan la cuenta de capitales.

En este estudio el objetivo fue demostrar la validez de la hipótesis nula: un tipo de cambio competitivo podría acelerar el crecimiento durante el período entre 1993-2018 caracterizado por estancamiento en la economía mexicana. En el análisis econométrico al comparar el TCRE estimado con el crecimiento económico, encontramos una fuerte desviación en la que el tipo de cambio se encuentra por debajo (depreciación) del TCRE estimado sin impulsar el crecimiento a partir del 2014. En los resultados de la estimación (ver Tabla 3) se observa que el coeficiente TCRE_desequilibrio (NEG) es significativo pero el impulso al crecimiento es muy bajo (0.017). Por otra parte, de los coeficientes TCRE_desequilibrio (POS) que se incluyen en el modelo sólo para el primer rezago es significativo y ejerce un efecto negativo, pero muy pequeño en el crecimiento que se disipa rápidamente. De hecho, los períodos de apreciación del peso mexicano son consecuencia de los problemas de la estructura y desequilibrio en la balanza de pagos en la economía. Es por ello que los flujos de capital necesarios para financiar el déficit en cuenta corriente ejercen el efecto de apreciar la moneda. En conclusión, se rechaza la hipótesis nula y por tanto un tipo de cambio competitivo no es la panacea del estancamiento productivo de México.

\section{Referencias}

Alexander, S.S. (1952), Effects of a devaluation on a Trade Balance, IMF Staff Papers, Núm. 2, pp. $263-278$.

Balassa, B., (1964), The purchasing power parity doctrine: A Reapraissal, Journal of Political Economy, núm. 72 , pp. 584-96.

Bai, J. y Perron P. (2003), Critical Values for Multiple Structural Change Tests, Econometrics Journal, 18, pp. 1-22. Bickerdike, C.F. (1920), The Instability of Foreign Exchange, Economic Journal, núm. 30, pp. 118-122.

Bresser-Pereira L. (2007), El Nuevo desarrollismo y la ortodoxia convencional, ECONOMIA-UNAM, Vol. 4, No. 10 , pp. 7-29.

_ (2011), "From old to new developmentalism in Latin America", Handbook of Latin America Economics, J.A. Ocampo y J. Ros (eds.), Oxford, Oxford University Press.

Clark, P.B. y MacDonald, R. (1998), Exchanges Rates and Economic Fundamentals: A Methodological Comparison of BEERs and FEERs, en MacDonald, R. y Stein J. (edits), Equilibrium Exchange Rates, London: Kluwer Academic Publishers, pp. 285-322.

Dvoskin A. y Feldman, G.D., 2015, Política cambiaria, distribución del ingreso y estructura productiva, en Estructura Productiva y Política Macroeconómica: Enfoques Heterodoxos desde América Latina, Ed. Bárcena A., Prado A. y Abeles M., CEPAL pp. 63-100.

Einzig, P. (1970), The History of Foreign Exchange, Macmillan.

Elbadawi, I.A., 1994, Estimating long-run equilibrium exchange rates, en Williamson, J. 
(Ed.), Estimating Equilibrium Exchange Rates, Institute for International Economics, Washington D.C., pp. $93-$ 132.

Engle, R. F., y C. W. J. Granger (1987), Co-integration and Error Correction: Representation, Estimation and Testing, Econometrica, 55, pp. 251-276.

Fleming, J.M. (1962), Domestic financial policies under fixed and under floating exchange rate, IMF Staff Papers, Núm.12, pp. 369-380.

Frenkel, J.A. (1981), Flexible Exchange Rate and International Macroeconomics, Chicago: University of Chicago Press.

Frenkel, R. (2006), An alternative to inflation targeting in Latin America: macroeconomics policies focus in unemployment, Journal of Post-Keynesian Economics, Vol. 28, No 4, pp. 573-591.

Frenkel, R. y Ros J. (2006), Unemployment and the real Exchange rate in Latin America, World Development, Vol. 34, Issue 4, pp. 631-646.

Friedman, M. (1953), The case for flexible exchange rates en Essays in Positive Economics. Chicago, Chicago University Press, pp. 157-203.

Harberger, A.C. (1950), Currency depreciation, Income and the Balance of Trade, Journal of Political Economy, Núm. 58, pp. 47-60.

Hicks, J.R. (1937), Mr. Keynes and "The Classics": A suggested interpretation, Econometrica, Vol. 5, pp.149-157.

Houthakker, H.S. y Magee, S.P. (1969), Income and price elasticities in world trade, Review of Economics and Statistics, 51, pp. 111-125.

Isard P. (1995), Exchange Rate Economics, Cambridge Surveys of Economic Literature

Keynes, J.M. (1923), A tract of Monetary Reform, London, Macmillan.

Krugman, P. (1989), Exchange rate instability, Cambridge Mass. MIT Press.

__(1990), Equilibrium Exchange Rates, in Branson et. al, editores, pp. 159-187.

Meade, J.E. (1951), The Theory of International Economic Policy, Vol. 1: The Balance of Payments, London: Oxford University Press.

Metzler, L.A. (1949), The Theory of International Trade, en Howard S. Ellis edit., A Survey of Contemporary Economics, Philadelphia: Blakiston, pp. 210-254.

Meese, R.A. y K. Rogoff (1983a), Empirical exchange rate models of the seventies: Do they fit out of the sample? Journal of International Economics, Núm. 14, pp. 3-24.

_ (1983b), The out-of-sample failure of empirical Exchange Rate Models: Sampling error or misspecification? en Exchange Rates and International Macroeconomics en J.A. Frenkel (Ed.), pp. 67-112.

Mundell, R.A. (1960), The monetary dynamics of International adjustment under fixed and floating exchange rates, Quarterly Journal of Economics, Núm. 74, pp. 227-257.

__(1961a), The international disequilibrium system, Kyklos 14, Fasc. 2, pp. 153-172.

_(1961b), A Theory of Optimum Currency Areas, American Economic Review, Núm. 51, pp. 657-665.

_ (1961c), Flexible Exchange Rate and Employment Policy, Canadian Journal of Economics and Political Science, Núm. 67, pp. 509-517.

__(1962), The appropriate use of monetary and fiscal policy for internal and external stability, IMF Staff Papers, Núm. 12, pp. 70-79.

__(1963), Capital mobility and stabilization policy under fixed and flexible Exchange Rates, Canadian Journal of Economics and Political Science, Núm. 29, pp. 475-485.

Nurkse, R. (1944), International Currency Experience: Lessons of the interwar period, Geneva: League of Nations.

Perrotini, H. I. y Vázquez, J.A. (2017), Endogenous growth and economic capacity: Theory and empirical evidence for the NAFTA countries, PSL Quarterly Review, vol. 70 n. $282 \quad$ septiembre, pp. 247-282.

Perrotini, I. y Vázquez, J.A. (2018), El súpermultiplicador, la acumulación de capital, las exportaciones y el crecimiento económico, EL TRIMESTRE ECONÓMICO, vol. LXXXV (2), núm. 338, abril-junio, pp. 411-432, http://dx.doi.org/10.20430/ete.v85i338.542 
Perrotini, I., Vázquez, J.A. y Angoa, I. (2019), Capital accumulation, economic growth and the balance-of-payments constraint: The case of Mexico, 1951-2014, Revista Noesis, Volumen 28, Núm. 55 enero - junio 2019. DOI http://dx.doi.org/10.20983/noesis.2019.1.3

Razmi, A., M. Rapetti y P. Skott (2012), The real exchange rates and economicdevelopment, structural Change and Economic Dynamics, vol. 23, № 2, pp. 151-169.

Robinson, J., (1947), The Foreign Exchanges, en Robinson Essay in The Theory of Employment, Oxford: Basil Blackwell, pp. 134-155.

Rodrik, D. (2008), The Real Exchange Rate and Economic Activity, Brookings Papers on Economic Activity, Harvard University.

Ros, J. y Galindo L.M., (2006), Banco de México: Política Monetaria de Metas de Inflación, ECONOMÍA-UNAM, Vol. 3, No 9 .

_, (2009), Alternatives to inflation targeting in México, en G.A. Epstein y A.E. Yeldan (Edits), Beyond inflation targeting: assessing the impacts and policy alternatives, E. Elgar, Chetelham.

_,(2013), Rethinking Economic Development, Growth and Institutions, Oxford, Oxford University Press.

Samuelson, P., (1964), Theoretical notes of Trade Problems, Review of Economics and Statistics, Núm. 46, pp. 145-154.

Williamson, J., (1985), The Exchange Rate System, Policy Analysis in International Economics, Núm. 5, Institute of International Economics, Washington DC.

_.,(1994), Estimating Equilibrium Exchange Rates, Institute of International Economics, Washington DC. 


\section{Anexo}

Tabla A1

Pruebas de Integración

\begin{tabular}{|c|c|c|c|c|c|c|}
\hline \multicolumn{7}{|c|}{ Prueba de raíz unitaria (Phillips-Perron) } \\
\hline Modelo & En nivel & TCRE & TOT & TNT & MON & RES \\
\hline \multirow[t]{2}{*}{ A } & t-Estadístico & -0.56 & -3.06 & -0.61 & 9.86 & -0.05 \\
\hline & Probabilidad & $0.87 * *$ & $0.03 * *$ & 0.86 & 1.00 & 0.95 \\
\hline \multirow[t]{2}{*}{ B } & t-Estadístico & -2.32 & -2.95 & -2.36 & 2.54 & -2.17 \\
\hline & Probabilidad & 0.42 & 0.15 & 0.40 & 1.00 & 0.50 \\
\hline \multirow[t]{2}{*}{$\mathrm{C}$} & t-Estadístico & 2.35 & 0.56 & 1.85 & 15.93 & 2.15 \\
\hline & Probabilidad & 1.00 & 0.84 & 0.98 & 1.00 & 0.99 \\
\hline Modelo & Primera diferencia & $\mathrm{d}$ (TCRE) & $\mathrm{d}(\mathrm{TOT})$ & $\mathrm{d}(\mathrm{TNT})$ & $\mathrm{d}(\mathrm{MON})$ & $\mathrm{d}(\mathrm{RES})$ \\
\hline \multirow[t]{2}{*}{ A } & t-Estadístico & -10.83 & -15.47 & -10.72 & -8.62 & -6.62 \\
\hline & Probabilidad & $0 * * *$ & $0 * * *$ & $0 * * *$ & $0 * * *$ & $0 * * *$ \\
\hline \multirow[t]{2}{*}{$\mathrm{B}$} & t-Estadístico & -10.77 & -14.45 & -10.66 & -10.23 & -6.61 \\
\hline & Probabilidad & $0 * * *$ & $0 * * *$ & $0 * * *$ & $0 * * *$ & $0 * * *$ \\
\hline \multirow[t]{2}{*}{$\mathrm{C}$} & t-Estadístico & -10.19 & -15.48 & -10.27 & -5.91 & -6.11 \\
\hline & Probabilidad & $0 * * *$ & $0 * * *$ & $0 * * *$ & $0 * * *$ & $0 * * *$ \\
\hline & \multicolumn{6}{|c|}{ Prueba de raíz unitaria Dickey-Fuller aumentada } \\
\hline Modelo & En nivel & TCRE & TOT & TNT & MON & RES \\
\hline \multirow[t]{2}{*}{ A } & t-Estadístico & -0.69 & -2.15 & -0.77 & 2.36 & -0.16 \\
\hline & Probabilidad & 0.84 & 0.23 & 0.82 & 1.00 & 0.94 \\
\hline \multirow[t]{2}{*}{ B } & t-Estadístico & -2.35 & -1.69 & -2.36 & 1.40 & -2.40 \\
\hline & Probabilidad & 0.40 & 0.75 & 0.40 & 1.00 & 0.38 \\
\hline \multirow[t]{2}{*}{$\mathrm{C}$} & t-Estadístico & 2.02 & 0.61 & 1.48 & 2.36 & 1.69 \\
\hline & Probabilidad & 0.99 & 0.85 & 0.97 & 1.00 & 0.98 \\
\hline Modelo & Primera diferencia & $\mathrm{d}$ (TCRE) & $\mathrm{d}(\mathrm{TOT})$ & $\mathrm{d}(\mathrm{TNT})$ & $\mathrm{d}(\mathrm{MON})$ & $\mathrm{d}(\mathrm{RES})$ \\
\hline \multirow[t]{2}{*}{ A } & t-Estadístico & -10.75 & -8.77 & -10.59 & -0.20 & -6.62 \\
\hline & Probabilidad & $0 * * *$ & $0 * * *$ & $0 * * *$ & 0.93 & $0 * * *$ \\
\hline \multirow[t]{2}{*}{$\mathrm{B}$} & t-Estadístico & -10.70 & -8.97 & -10.54 & -1.94 & -6.61 \\
\hline & Probabilidad & $0 * * *$ & $0 * * *$ & $0 * * *$ & 0.62 & $0 * * *$ \\
\hline \multirow[t]{2}{*}{$\mathrm{C}$} & t-Estadístico & -10.19 & -8.75 & -10.27 & 1.16 & -6.11 \\
\hline & Probabilidad & $0 * * *$ & $0 * * *$ & $0 * * *$ & 0.94 & $0 * * *$ \\
\hline
\end{tabular}

Nota: Nivel de significancia al $* 0.1, * * 0.05, * * * 0.01$; los estadísticos en negritas indican que no son significativas A, B y C corresponden a los modelos con constante, con constante y tendencia y sin constante y tendencia, respectivamente.

Fuente: Elaboración propia. 
Tabla A2

Prueba de cointegración Engel-Granger

H0: Series no cointegradas

\begin{tabular}{lccc}
\hline & Valor & Probabilidad \\
\hline Engle-Granger tau-statistic & -4.70 & 0.04 & \\
Engle-Granger z-statistic & -33.92 & 0.05 & \\
Nota: MacKinnon (1996) p-values. & & & \\
\hline \multicolumn{5}{c}{ Prueba Hansen de estabilidad en los parámetros } \\
Estocástica & Determinista & Excluida & \\
H0: Series cointegradas & Trends (k) & Trends (p2) & Prob.* \\
\hline Trends (m) & 0 & 0 & $>0.2$ \\
\hline
\end{tabular}

Nota: la probabilidad corresponde al $10 \%$ de significancia.

Fuente: elaboración propia.

Tabla A3

Pruebas de correlación serial y heterocedasticidad

\begin{tabular}{lcc}
\hline Breusch-Godfrey Serial Correlation LM Test: & Estadístico F & Prob. Chi-Square \\
\hline Hipótesis Nula: No hay correlación serial & & \\
\hline arriba de 1 rezago & 0.39 & 0.50 \\
arriba de 2 rezagos & 0.45 & 0.59 \\
arriba de 3 rezagos & 0.41 & 0.75 \\
arriba de 4 rezagos & F-statistic & Prob. Chi-Square(10) \\
\hline Prueba Breusch-Pagan de Heterocedasticidad & 1.35 & 0.21 \\
\hline Hipótesis Nula: Homocedasticidad & F-statistic & Prob. Chi-Square(1) \\
\hline Prueba Heteroscedasticidad: ARCH & 0.00 & 0.94 \\
\hline
\end{tabular}

Nota: Nivel de significancia al 5\%.

Fuente: Elaboración propia. 